\title{
Serum levels of vascular endothelial growth factor in non-alcoholic fatty liver disease
}

\section{Maria-Vasiliki Papageorgioua , Emilia Hadziyannis ${ }^{b}$, Dina Tiniakosc, Anastasia Georgioua , Aikaterini Margariti ${ }^{b}$, Athanasios Kostas ${ }^{a}$, George V. Papatheodoridis ${ }^{a}$}

Laiko General Hospital, Athens, Greece; Hippokration General Hospital, Athens, Greece; Medical School, National and Kapodistrian University of Athens, Greece

\section{Abstract}

${ }^{a}$ Academic Department of Gastroenterology, Medical School, National and Kapodistrian University of Athens, Laiko General Hospital (MariaVasiliki Papageorgiou, Anastasia Georgiou, Athanasios Kostas, George V. Papatheodoridis); ' 2 ndAcademic Department of Internal Medicine, Medical School, National and Kapodistrian University of Athens, Hippokration General Hospital (Emilia Hadziyannis, Aikaterini Margariti); 'Laboratory of Histology \& Embryology, Medical School, National and Kapodistrian University of Athens (Dina Tiniakos), Athens, Greece

Conflict of interest: None

Correspondence to: George V. Papatheodoridis, MD, PhD, Academic Department of Gastroenterology, Laiko General Hospital of Athens, 17 Agiou Thoma St., 11527 Athens, Greece, Tel.: +30 2132061115, Fax: +30 2107462601, e-mail: gepapath@med.uoa.gr

Received 06 September 2016; accepted 25 October 2016; published online 01 December 2016

DOI: https://doi.org/10.20524/aog.2016.0107

\section{Introduction}

Non-alcoholic fatty liver disease (NAFLD) represents a major public health problem with a prevalence as high as $30 \%$ in many populations $[1,2]$. Liver biopsy is considered to be the gold standard for the assessment of the presence and severity of hepatic injury in NAFLD, but it is an invasive procedure that has also been associated with sampling errors $[3,4]$. Therefore, there is growing scientific interest in imaging studies and serum-based assays that aim to detect the presence of NAFLD, and to distinguish simple fatty liver (FL) from non-alcoholic steatohepatitis (NASH), the more severe and progressive type of NAFLD [5].

Considering that NASH is characterized by marked activation of inflammatory cells [6-9] and upregulation of several soluble inflammatory mediators, the role of different 
cytokines and chemokines in NAFLD has been evaluated by several groups [10]. Liver inflammation, which is a key element of NASH, has been shown to be triggered by activated cytokines induced by lipotoxicity. Although the pathogenesis of NASH is still not fully understood, some studies suggested that angiogenesis might play a role in its progression $[11,12]$. Inflammatory cells are able to initiate angiogenesis through different pathways, thereby contributing to the formation of new vasculature in the liver $[13,14]$. Although angiogenesis has been well documented in patients with chronic viral hepatitis [15,16], information regarding angiogenesis in NAFLD is very limited.

Vascular endothelial growth factor (VEGF) is a proangiogenic factor implicated in the angiogenetic process. The aim of this study was to evaluate the possible significance of serum VEGF levels in patients with NAFLD.

\section{Patients and methods}

Sixty-seven consecutive patients with NAFLD who visited our outpatient liver clinics between May 2008 and December 2010 were included. The diagnosis of NAFLD was based on the following criteria: elevated alanine aminotransferase (ALT) and/or gamma-glutamyl transpeptidase (GGT) levels; evidence of hepatic steatosis on ultrasonography and/or liver histology; and exclusion of other causes of liver injury. In particular, all patients had negative serological markers for hepatitis B (HBsAg), hepatitis C (anti-HCV) and human immunodeficiency virus (anti-HIV), weekly alcohol consumption less than $210 \mathrm{~g}$ for men or $140 \mathrm{~g}$ for women, no use of potentially hepatotoxic agents, no evidence of metabolic or autoimmune liver disease, and absence of any known systemic disease with potential liver involvement. The history of alcohol use was taken from the patients and was confirmed by the patients' relatives or friends.

Demographic characteristics and medical history were recorded for all patients, together with any history of known arterial hypertension or the presence of diabetes mellitus, diagnosed on the basis of antidiabetic treatment and/or fasting glucose $>126 \mathrm{mg} / \mathrm{dL}$ on more than one occasion.

Forty-seven healthy controls matched for age, sex and body mass index (BMI) with 47 of the aforementioned 67 patients were also enrolled. Controls were either subjects who visited the outpatient clinics of our hospital for routine examinations during the same period, or hospital staff members. Controls had normal glucose metabolism and liver biochemistry and no evidence of hepatic steatosis on abdominal ultrasound.

The study was approved by the Hippokratio Hospital Institutional Review Board.

\section{Anthropometric assessments}

Participants' body weight was measured with a digital scale (Seca Robusta 813, Hamburg, Germany) to the nearest
$100 \mathrm{~g}$ and height was measured to the nearest $0.5 \mathrm{~cm}$. Waist circumference $(\mathrm{WC})$ was tape-measured to the nearest $0.1 \mathrm{~cm}$. Increased WC was defined as $>102 \mathrm{~cm}$ for men and $>88 \mathrm{~cm}$ for women.

\section{Laboratory markers}

The laboratory data recorded included complete blood count, prothrombin time, urea, creatinine, urate, liver enzymes (ALT, aspartate aminotransferase, alkaline phosphatase, and GGT), total protein, albumin, as well as detection of $\mathrm{HBsAg}$, anti-HBc, anti-HBs, anti-HCV, anti-HIV, and liver autoantibodies.

Serum VEGF levels were measured using a commercially available sensitive enzyme-linked immunosorbent assay (ELISA, Quantikine/immunoassay kit, R\&D Systems, Minneapolis, MN, USA). The intra- and inter-assay coefficients of variation were $<7 \%$ and $<10 \%$, respectively. Serum levels of caspase-generated fragments of keratin-18 (K18) as well as of and soluble Fas (sFas) were also measured by ELISA based immunoassays (M30-Apoptosense ELISA assay, PEVIVA, Alexis, Grünwald, Germany and Human Fas/TNFRSF6 Quantikine ELISA Kit, R\&D Systems, Minneapolis, MN, USA, respectively).

\section{Definitions}

Patients were considered obese if they had a BMI $\geq 30 \mathrm{~kg} / \mathrm{m}^{2}$. Metabolic syndrome was defined according to the National Cholesterol Education Program: Adult Treatment Panel III criteria [17].

Patients with NAFLD were classified into those with FL and those with NASH, according to the widely accepted histological criteria described below and/or to a recently published formula based on serum K18 and sFas levels, shown to correctly classify $88 \%$ of NAFLD patients [18].

\section{Transient elastography (TE)}

Liver stiffness was measured (in $\mathrm{kPa}$ ) using the standard probe of TE (Fibroscan, Echosens, France) in 48 of the 67 patients, but the results were reliable in only 46 . The result was considered reliable if 10 successful measurements were obtained, with a success ratio $>60 \%$ and a ratio of interquartile range to mean stiffness $<30 \%$. For patients who underwent both TE and liver biopsy, liver stiffness measurement was performed a few hours before liver biopsy in most or within 4 weeks before or after liver biopsy in some patients.

\section{Liver histology}

Adequate liver biopsies were obtained in 34 of the 67 NAFLD patients. Histological lesions were classified according 
to the Brunt classification by one blinded liver histopathologist (DT). A liver biopsy was considered to be adequate if at least 6 portal tracts were identified and the specimen length was $\geq 1.5 \mathrm{~cm}$. The diagnosis of NASH was made according to the Brunt classification criteria [19], as modified by Kleiner et al [20]. Global grading of necroinflammatory activity and staging of fibrosis were assessed according to Brunt et al [19]. Severity of steatosis and NAFLD activity score were evaluated according to Kleiner et al [20].

\section{Statistical analysis}

Quantitative variables with normal distribution were expressed as mean values \pm standard deviation (SD) and those with abnormal distribution as median values (range). Statistical analysis was performed using the Mann-Whitney test for comparisons of quantitative variables between groups, Spearman's coefficient for correlations of quantitative variables, and a two-tailed Fisher's exact test for qualitative data. The accuracy of VEGF levels for predicting early (stage: $0-1$ ) or advanced (stage: 2-4) liver disease was assessed by the area under the receiver operating characteristic curve (AUROC). A two-tailed P-value of $<0.05$ was considered to be statistically significant.

\section{Results}

Of the 67 NAFLD patients, 21 (31\%) were diagnosed with FL and 46 (69\%) with NASH. FL and NASH were diagnosed in $11(32 \%)$ and $23(68 \%)$ of the 34 patients who underwent liver biopsy, and in $10(30 \%)$ and $23(70 \%)$ of the remaining 33 patients without a liver biopsy according to the K-18/sFas formula. In the 34 patients with a liver biopsy, the accuracy of the K-18/sFas formula in differentiating between the histological presence of FL and NASH was $91 \%(31 / 34)$.

Baseline patient characteristics are shown in Table 1. Patients with NASH had higher WC $(107 \pm 10$ vs. $103 \pm 13 \mathrm{~cm}, \mathrm{P}=0.016)$

Table 1 Demographic, anthropometric, clinical and laboratory characteristics of the study population

\begin{tabular}{|c|c|c|c|c|c|c|}
\hline Characteristic & $\begin{array}{l}\text { Patients with } \\
\text { fatty liver } \\
\quad(n=21)\end{array}$ & $\begin{array}{c}\text { Patients } \\
\text { with NASH } \\
\quad(\mathrm{n}=46)\end{array}$ & $\mathrm{P}$ & $\begin{array}{l}\text { Matched patients } \\
\text { with NAFLD } \\
\quad(\mathrm{n}=47)\end{array}$ & $\begin{array}{l}\text { Healthy controls } \\
\qquad(\mathrm{n}=47)\end{array}$ & $\mathrm{P}$ \\
\hline Age, years & $43 \pm 9$ & $44 \pm 11$ & 0.425 & $46 \pm 12$ & $47 \pm 13$ & 0.777 \\
\hline Sex, males, n (\%) & $11(52)$ & $34(74)$ & 0.099 & $27(57)$ & $27(57)$ & $>0.999$ \\
\hline Body mass index, $\mathrm{kg} / \mathrm{m}^{2}$ & $27 \pm 3$ & $30 \pm 4$ & 0.060 & $27 \pm 4$ & $28 \pm 4$ & 0.054 \\
\hline Waist circumference, $\mathrm{cm}$ & $99 \pm 7$ & $106 \pm 10$ & 0.016 & $104 \pm 10$ & $100 \pm 10$ & 0.097 \\
\hline Smoking, n (\%) & & & 0.229 & & & 0.046 \\
\hline Current & $3(62)$ & $14(30)$ & & $10(22)$ & $20(43)$ & \\
\hline Never & 13 & 21 & & $26(57)$ & $16(35)$ & \\
\hline History & 5 & 11 & & $11(24)$ & $11(24)$ & \\
\hline Concomitant diseases, $\mathrm{n}(\%)$ & & & 0.426 & & & 0.007 \\
\hline Diabetes, n (\%) & $1(5)$ & $0(0)$ & & $1(2)$ & $1(2)$ & \\
\hline Arterial hypertension, $\mathrm{n}(\%)$ & $0(0)$ & $2(4)$ & & $2(4)$ & $5(11)$ & \\
\hline Coronary artery disease, $\mathrm{n}(\%)$ & $1(5)$ & $0(0)$ & & $0(0)$ & $1(2)$ & \\
\hline Dyslipidemia, n (\%) & $7(33)$ & $22(48)$ & & $22(47)$ & $2(4)$ & \\
\hline Other, n (\%) & $1(5)$ & $1(5)$ & & $4(9)$ & $6(13)$ & \\
\hline ALT, IU/L $(\mathrm{ULN}=40)$ & $61 \pm 29$ & $69 \pm 30$ & 0.342 & $73 \pm 33$ & $31 \pm 19$ & $<0.001$ \\
\hline AST, IU/L $(\mathrm{ULN}=40)$ & $35 \pm 15$ & $39 \pm 13$ & 0.234 & $44 \pm 21$ & $23 \pm 6$ & $<0.001$ \\
\hline GGT, U/L (ULN=50) & $72(16-168)$ & $39(21-753)$ & 0.072 & $45(17-753)$ & $18(5-58)$ & $<0.001$ \\
\hline Cholesterol, mg/dL - Total & $239 \pm 51$ & $209 \pm 41$ & 0.053 & $222 \pm 47$ & $246 \pm 35$ & 0.106 \\
\hline - HDL & $50 \pm 12$ & $43 \pm 9$ & 0.060 & $48 \pm 12$ & $66 \pm 28$ & 0.227 \\
\hline - LDL & $154 \pm 34$ & $133 \pm 31$ & 0.151 & $142 \pm 33$ & $154 \pm 43$ & 0.425 \\
\hline Triglycerides, mg/dL & $155 \pm 121$ & $192 \pm 86$ & 0.069 & $159 \pm 76$ & $131 \pm 103$ & 0.283 \\
\hline
\end{tabular}

${ }^{*}$ Patients with NAFLD matched to healthy controls for age, gender and body mass index

NASH, non-alcoholic steatohepatitis; NAFLD, non-alcoholic fatty liver disease; ULN, upper limit of normal; ALT, alanine aminotransferase; AST, aspartate aminotransferase; GGT, gamma-glutamyl transpeptidase; HDL, high-density lipoprotein; LDL, low-density lipoprotein 
compared to patients with FL. Though the differences were not statistically significant, NASH patients also tended to have higher BMI ( $30 \pm 4$ vs. $29 \pm 5 \mathrm{~kg} / \mathrm{m}^{2}, \mathrm{P}=0.06$ ), higher triglyceride levels ( $175 \pm 75$ vs. $155 \pm 104 \mathrm{mg} / \mathrm{dL}, \mathrm{P}=0.069)$ and lower HDL levels ( $44 \pm 10$ vs. $51 \pm 13 \mathrm{mg} / \mathrm{dL}, \mathrm{P}=0.060$ ) compared to those with FL.

In the 47 NAFLD patients who were matched to healthy controls, VEGF levels were lower in patients than in controls ( $296 \pm 146$ vs. $365 \pm 186 \mathrm{pg} / \mathrm{mL}$ ) but the difference did not reach statistical significance $(\mathrm{P}=0.092)$. Given that no difference in the VEGF levels was found between patients with FL and controls ( $336 \pm 136$ vs. $365 \pm 186 \mathrm{pg} / \mathrm{mL}, \mathrm{P}=0.532)$, the previous finding was attributed to patients with NASH. In particular, patients with NASH had significantly lower VEGF levels compared to healthy subjects $(279 \pm 149$ vs. $365 \pm 186 \mathrm{pg} / \mathrm{mL}$,

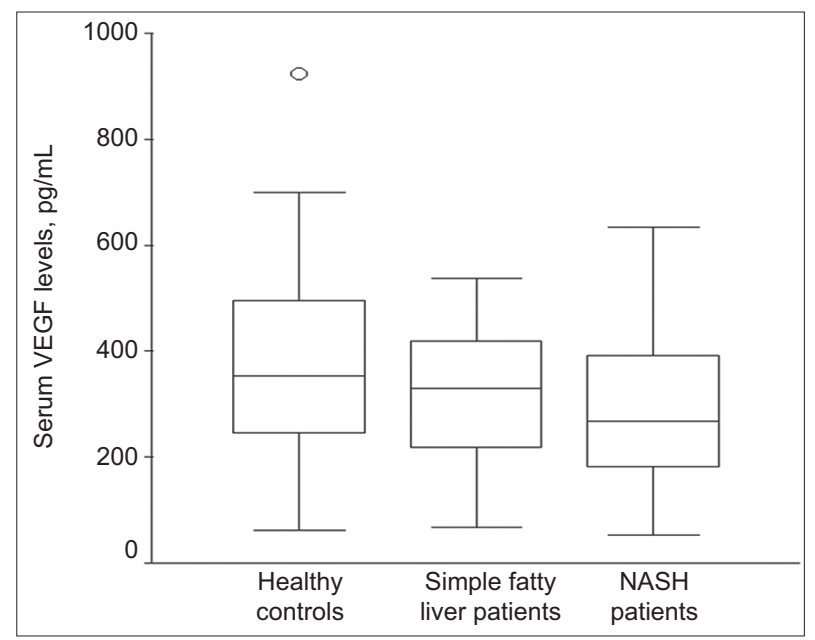

Figure 1 Serum levels of vascular endothelial growth factor (VEGF) in healthy controls and in patients with simple fatty liver or non-alcoholic steatohepatitis (NASH)

Controls vs. NASH: $\mathrm{P}=0.041$, fatty liver vs. NASH: $\mathrm{P}=0.095$. Box and whisker plots express medians, and interquartile and overall ranges. The outlying values are plotted individually
$\mathrm{P}=0.041$ ). In addition, serum VEGF levels tended to be lower in the 46 patients with NASH compared to the 21 patients with FL $(289 \pm 147$ vs. $359 \pm 190 \mathrm{pg} / \mathrm{mL}, \mathrm{P}=0.095)$ (Fig. 1). A similar trend in serum VEGF levels was also observed in the 34 patients who underwent liver biopsy (FL: $326 \pm 130$ vs. NASH: $253 \pm 149 \mathrm{pg} / \mathrm{mL}, \mathrm{P}=0.098$ ). These data suggest that serum VEGF levels tend to decrease with the progression from simple steatosis to steatohepatitis.

Serum VEGF levels were not able to differentiate effectively between NAFLD patients and controls (AUROC: 0.601, 95\% CI 0.486-0.715; $\mathrm{P}=0.092$ ) (Fig. 2A). In the 67 NAFLD patients, serum VEGF levels also could not differentiate between FL and NASH patients (AUROC: 0.628, 95\% CI 0.485-0.770; $\mathrm{P}=0.095$ ) (Fig. 2B). In the 34 NAFLD patients with liver biopsy, VEGF levels were numerically but not statistically higher in patients with an early stage of fibrosis (0-1) than in those with an advanced stage (2-4) $(302 \pm 141$ vs. $239 \pm 149 \mathrm{pg} / \mathrm{mL}, \mathrm{P}=0.129)$, while there was no significant correlation between VEGF levels and fibrosis stage $(\mathrm{r}=-0.268, \mathrm{P}=0.125)$.

When we split our NAFLD patients according to their median VEGF value, patients with high ( $\geq 300 \mathrm{pg} / \mathrm{mL}$ ) VEGF levels were found to have FL significantly more frequently, both in the total NAFLD population ( $67 \%$ vs. $35 \%, \mathrm{P}=0.019)$ and in the 34 NAFLD patients with liver biopsy (57\% vs. $15 \%$, $\mathrm{P}=0.023)$. Patients with high VEGF levels also had a lower mean fibrosis score $(0.7 \pm 0.9$ vs. $1.6 \pm 1.0, \mathrm{P}=0.017)$ (Table 2$)$.

No significant correlation was observed between serum VEGF levels and age $(\mathrm{r}=0.104, \mathrm{P}=0.271)$, BMI $(\mathrm{r}=-0.109$, $\mathrm{P}=0.247)$, sex $(\mathrm{r}=0.090, \mathrm{P}=0.342)$, liver inflammation expressed by histological grading $(\mathrm{r}=-0,116, \mathrm{P}=0.513)$, hepatic steatosis $(\mathrm{r}=0.182, \mathrm{P}=0.319)$ or liver stiffness at elastography $(\mathrm{r}=-0.117$, $\mathrm{P}=0.430$ ). Additionally, no significant correlation was found between serum VEGF levels and cholesterol $(\mathrm{r}=0.176$, $\mathrm{P}=0.526)$, triglyceride $(\mathrm{r}=0.064, \mathrm{P}=0.616)$ or $\mathrm{GGT}$ values $(\mathrm{r}=0.064, \mathrm{P}=0.526)$. Interestingly, serum VEGF levels had a significant inverse correlation with aspartate aminotransferase $(\mathrm{r}=-0.262, \mathrm{P}=0.007)$ and ALT values $(\mathrm{r}=-0.275, \mathrm{P}=0.005)$.
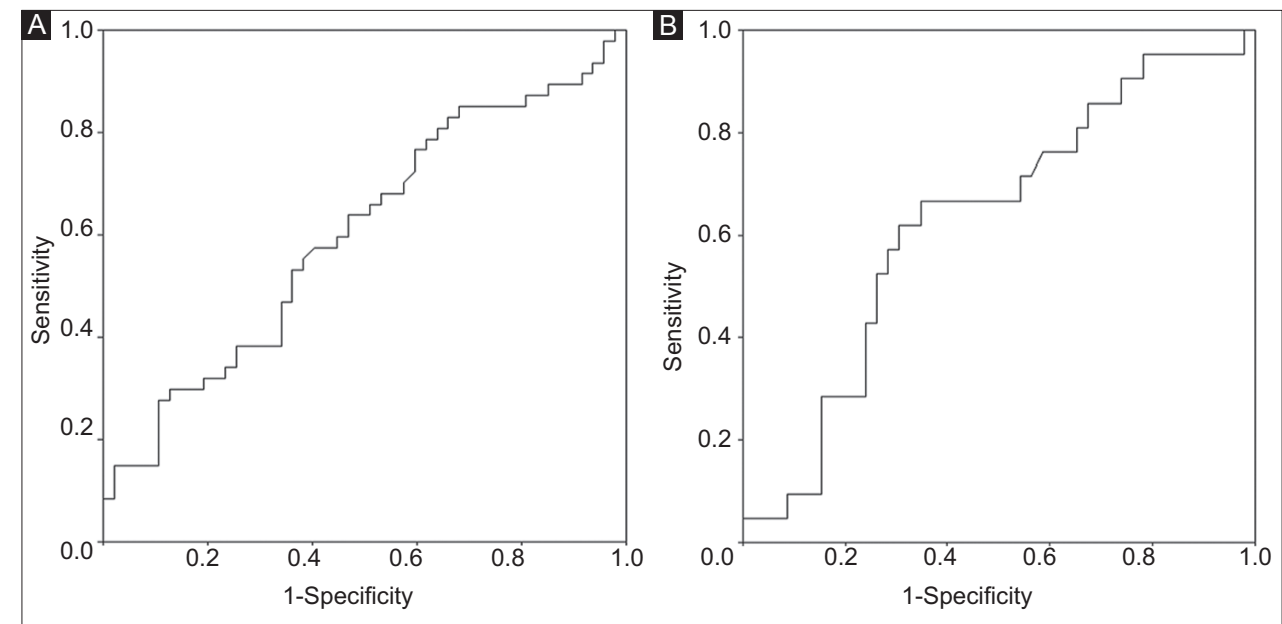

Figure 2 (A) Area under the receiving operating characteristic curve (AUROC) of serum levels of vascular endothelial growth factor for the discrimination between patients with non-alcoholic fatty liver disease and healthy controls (AUROC: $0.601,95 \% \mathrm{CI} 0.486-0.715$; $\mathrm{P}=0.092$ ) (B) or between patients with simple fatty liver and those with non-alcoholic steatohepatitis (AUROC: $0.628,95 \%$ CI $0.485-0.770 ; \mathrm{P}=0.095$ ) 
Table 2 Vascular endothelial growth factor (VEGF) serum levels in patients with non-alcoholic fatty liver disease (NAFLD) in relation to their characteristics

\begin{tabular}{|c|c|c|c|}
\hline Characteristic & $\begin{array}{l}\text { High VEGF }(\geq 300 \mathrm{pg} / \mathrm{mL}) \\
\qquad(\mathrm{n}=30)\end{array}$ & $\begin{array}{l}\text { Low VEGF }(<300 \mathrm{pg} / \mathrm{mL}) \\
\qquad(\mathrm{n}=37)\end{array}$ & $\mathrm{P}$ \\
\hline Age, years & $44 \pm 10$ & $46 \pm 12$ & 0.566 \\
\hline Sex, males (\%) & $18(60)$ & $27(73)$ & 0.303 \\
\hline Body mass index, $\mathrm{kg} / \mathrm{m}^{2}$ & $29 \pm 4$ & $31 \pm 4$ & 0.221 \\
\hline Waist circumference, $\mathrm{cm}$ & $105 \pm 10$ & $108 \pm 12$ & 0.549 \\
\hline Smoking, n (\%) & & & 0.056 \\
\hline Current & $9(30)$ & $8(22)$ & \\
\hline Never & $18(60)$ & $16(43)$ & \\
\hline History & $3(10)$ & $13(35)$ & \\
\hline Concomitant diseases, $\mathrm{n}(\%)$ & & & 0.492 \\
\hline None & $15(50)$ & $14(38)$ & \\
\hline Diabetes & $1(3)$ & $0(0)$ & \\
\hline Arterial hypertension & $0(0)$ & $2(5)$ & \\
\hline Coronary artery disease & $0(0)$ & $1(3)$ & \\
\hline Dyslipidemia & $12(40)$ & $17(46)$ & \\
\hline Other & $2(7)$ & $3(8)$ & \\
\hline ALT, IU/L (ULN=40) & $56 \pm 26$ & $74 \pm 30$ & 0.078 \\
\hline AST, IU/L (ULN=40) & $35 \pm 15$ & $40 \pm 13$ & 0.245 \\
\hline GGT, U/L (ULN=50) & $91 \pm 77$ & $89 \pm 37$ & 0.113 \\
\hline Cholesterol, mg/dL - Total & $224 \pm 47$ & $214 \pm 45$ & 0.112 \\
\hline - HDL & $50 \pm 11$ & $43 \pm 10$ & 0.066 \\
\hline - LDL & $142 \pm 34$ & $137 \pm 34$ & 0.589 \\
\hline Triglycerides, mg/dL & $161 \pm 86$ & $195 \pm 104$ & 0.390 \\
\hline Liver stiffness by elastography, $\mathrm{kPa}$ & $6 \pm 2$ & $8 \pm 6$ & 0.150 \\
\hline NAFLD type, n (\%) & & & 0.019 \\
\hline Simple fatty liver & $14(67)$ & $16(35)$ & \\
\hline Non-alcoholic steatohepatitis & $7(33)$ & $30(65)$ & \\
\hline NAFLD type according to liver biopsy, $\mathrm{n}(\%)$ & & & 0.023 \\
\hline Simple fatty liver & $8(57)$ & $3(15)$ & \\
\hline Non-alcoholic steatohepatitis & $6(43)$ & $17(85)$ & \\
\hline Histological grading & $0.7 \pm 0.8$ & $1.1 \pm 0.87$ & 0.180 \\
\hline Fibrosis score & $0.7 \pm 0.9$ & $1.6 \pm 1.0$ & 0.017 \\
\hline Severity of fibrosis, n (\%) & & & 0.079 \\
\hline Stage $0-1$ & $11(79)$ & $9(45)$ & \\
\hline Stage $2-4$ & $3(21)$ & $11(55)$ & \\
\hline Steatosis score & $1.7 \pm 0.9$ & $1.8 \pm 0.9$ & 0.112 \\
\hline
\end{tabular}

ALT, alanine aminotransferase; AST, aspartate aminotransferase; GGT, gamma-glutamyl transpeptidase; ULN, upper limit of normal; HDL, high-density lipoprotein; LDL, low-density lipoprotein

\section{Discussion}

Like all chronic liver diseases, NAFLD is characterized by a wide spectrum of inflammation and fibrosis. NASH, the most severe form of NAFLD, can progress to cirrhosis and may eventually lead to hepatocellular carcinoma [21-23]. The twohit hypothesis, based on the initial intrahepatic accumulation of triglycerides followed at a second stage by inflammatory 
mediators, gut endotoxin, mitochondrial dysfunction and endoplasmic reticulum stress causing liver injury, has provided some explanation for the pathogenesis of NASH, but has recently been challenged. Currently, the pathophysiology of NAFLD is considered to be multifactorial, including a relation between changes in microvasculature and the progression of fibrosis [24-27]. Tissue damage from both fat accumulation and lipotoxicity results in reduced sinusoidal perfusion and changes in sinusoidal architecture. Additionally, several cytokines activated by lipotoxicity further induce the migration of inflammatory cells, which contribute to angiogenesis in the inflammatory foci [28]. During disease progression, fatty hepatocytes, tissue inflammation and perisinusoidal fibrosis constrict the sinusoidal lumens and impair the sinusoidal perfusion, leading to hypoxia [28,29]. All these events provoking liver damage can initiate angiogenesis [26]. As a consequence, angiogenesis leading to new vasculature formation may have prognostic value in disease progression. The idea that interfering with angiogenesis might be a potential target to avoid progression of liver disease has stimulated research into several markers for angiogenesis in chronic liver diseases.

Angiogenesis has been documented in cases of viral hepatitis, but information regarding angiogenesis in NAFLD is very limited. Angiogenesis in chronic hepatitis $\mathrm{C}$ has been reported to be induced more frequently than in patients with chronic hepatitis B or healthy controls [30]. Salcedo et al concluded that serum VEGF, angiopoietin 2 and tyrosine kinase 2 levels could be useful as noninvasive markers of response to therapy and disease progression in patients with chronic hepatitis C [31]. Moreover, enhanced angiogenesis has been described in association with hepatocellular carcinoma in patients with chronic hepatitis and cirrhosis [32,33]. In our study, VEGF serum levels did not differ between patients with $\mathrm{FL}$ and healthy controls, but were found to be lower in NASH patients compared to healthy controls $(\mathrm{P}=0.041)$ and tended to be lower in patients with NASH compared to those with FL $(\mathrm{P}=0.098)$. These findings suggest that serum VEGF levels tend to decrease during the progression from healthy liver or simple steatosis to steatohepatitis.

Most of the data on angiogenesis in NAFLD come from animal models, while the few reports on serum VEGF levels in NAFLD patients have been controversial. Kitade et al showed that angiogenesis is involved in the development of NASHrelated liver fibrosis and carcinogenesis in leptin-deficient rats [34]. In a rat model, it was also shown that renin inhibition may have favorable effects on liver fibrogenesis in NASH, through inhibition of angiotensin-II, tumor growth factor $\beta$ and VEGF [35]. More recently, Yang et al suggested that antiVEGF receptor agents ameliorate hepatic venous dysregulation, microcirculatory dysfunction, splanchnic venous pooling and ascites in NASH cirrhotic rats [36]. In humans, Amarapurkar et al reported that immunohistochemical VEGF hepatic expression was seen in $29 \%$ of NASH patients or $46 \%$ of patients with chronic liver disease of various etiologies, being more common in the early stages of fibrosis [37]. Yilmaz et al reported no significant difference in serum VEGF levels, but significantly lower serum levels of soluble VEGF receptor 1 in NAFLD patients compared to healthy controls, with lower soluble VEGF receptor 1 levels being associated with increased fibrosis [38]. On the other hand, Coulon et al found that serum VEGF serum levels were significantly higher in patients with FL compared to healthy controls, but only relatively higher in patients with NASH compared to healthy controls. In the same study, the concentration of soluble VEGF receptor 1 was significantly higher in the serum of FL and NASH patients compared to controls [39]. Moreover, Tarantino et al showed that serum VEGF levels were higher in NASH patients compared to patients with FL or healthy controls, but serum VEGF level was not a useful marker for differentiation between FL and NASH patients [40]. More recently, CiupinskaKajor et al reported that the immunohistochemical hepatic expression of VEGF was higher in simple steatosis and borderline NASH in severely obese patients and in NASH in non-obese patients with NAFLD [41]. In NASH, centrilobular arteries and increased microvessel density are more commonly detected in advanced fibrotic stages, suggesting a possible association between neoangiogenesis and NASH progression to cirrhosis [42].

In addition to the existing controversial data, our results also suggest that serum VEGF levels cannot accurately differentiate healthy controls from NAFLD patients, or patients with simple fatty liver from those with NASH. However, our finding of higher serum VEGF levels in healthy controls, or even perhaps in patients with FL, compared to those with NASH may seem strange, since increased angiogenesis is usually thought to be present in more advanced liver disease, as mentioned above. One explanation for such discrepant findings may be the type of assay used to determine serum VEGF levels. Only free VEGF was measured in our study, which means that the detected VEGF levels may, at least in some cases, underestimate the circulating VEGF levels in serum. In addition, we neither assessed the hepatic expression of VEGF nor measured VEGF receptors, which may offer important information about the role of VEGF in the pathogenesis of NAFLD. Another reason for these conflicting results could be the heterogeneity of patients and controls among the different studies. Finally, angiogenesis can be useful early in the course of NAFLD, reflecting the autohealing ability of the liver. However, beyond a critical point, angiogenesis may become injurious itself and VEGF inhibitors could predominate, thus resulting in a reduction in circulating VEGF levels. Alternatively, our results could reflect a possible dichotomous effect of VEGF on NAFLD progression, similar to that reported for adipose tissue dysfunction [43].

In conclusion, serum VEGF levels offer poor predictability in differentiating healthy controls from NAFLD patients or patients with FL from those with NASH. However, our data suggest that serum VEGF levels are similarly high in healthy controls and patients with FL and tend to decrease when NASH develops. This is a challenging finding, because it may indicate a dichotomous effect of VEGF levels in NAFLD; this question needs to be further evaluated in larger studies in order to clarify the role of VEGF in the pathogenesis of NAFLD. 


\section{Summary Box}

\section{What is already known:}

- Non-alcoholic steatohepatitis (NASH) is characterized by marked elevation of inflammatory cells

- Angiogenesis might play a role in the progression of NASH

- Vascular endothelial growth factor (VEGF) is a proangiogenic factor implicated in the angiogenetic process

- In the human setting, the few reports on serum VEGF levels in patients with non-alcoholic fatty liver disease (NAFLD) are controversial

\section{What the new findings are:}

- VEGF levels cannot reliably differentiate between patients with NAFLD and healthy controls or between patients with simple fatty liver (FL) and NASH

- Serum levels of VEGF are lower in patients with NASH than in healthy controls and relatively lower in NASH than in patients with FL

- NAFLD patients with high VEGF levels $(\geq 300 \mathrm{pg} / \mathrm{mL}$ ) have a lower incidence of NASH and a lower mean fibrosis score

\section{References}

1. Harrison SA, Torgerson S, Hayashi PH. The natural history of nonalcoholic fatty liver disease: a clinical histopathological study. Am J Gastroenterol 2003;98:2042-2047.

2. Satapathy SK, Sanyal AJ. Epidemiology and natural history of nonalcoholic fatty liver disease. Semin Liver Dis 2015;35:221-235.

3. Nalbantoglu IL, Brunt EM. Role of liver biopsy in nonalcoholic fatty liver disease. World J Gastroenterol 2014;20:9026-9037.

4. Ratziu V, Charlotte F, Heurtier A, et al; LIDO Study Group. Sampling variability of liver biopsy in nonalcoholic fatty liver disease. Gastroenterology 2005;128:1898-1906.

5. Hernaez R, Lazo M, Bonekamp S, et al. Diagnostic accuracy and reliability of ultrasonography for the detection of fatty liver: a meta-analysis. Hepatology 2011;54:1082-1090.

6. Hübscher SG. Histological assessment of non-alcoholic fatty liver disease. Histopathology 2006;49:450-465.

7. Tiniakos DG. Nonalcoholic fatty liver disease/nonalcoholic steatohepatitis: histological diagnostic criteria and scoring systems. Eur J Gastroenterol Hepatol 2010;22:643-650.

8. Brunt EM. Nonalcoholic steatohepatitis: pathologic features and differential diagnosis. Semin Diagn Pathol 2005;22:330-338.

9. Brunt EM, Kleiner DE, Wilson LA, et al; NASH Clinical Research Network. Portal chronic inflammation in nonalcoholic fatty liver disease (NAFLD): a histologic marker of advanced NAFLD-Clinicopathologic correlations from the nonalcoholic steatohepatitis clinical research network. Hepatology 2009;49:809820 .
10. Braunersreuther V, Viviani GL, Mach F, Montecucco F. Role of cytokines and chemokines in non-alcoholic fatty liver disease. World J Gastroenterol 2012;18:727-735.

11. Cayón A, Crespo J, Guerra AR, Pons-Romero F. [Gene expression in obese patients with non-alcoholic steatohepatitis]. Rev Esp Enferm Dig 2008;100:212-218.

12. Kitade M, Yoshiji H, Noguchi R, et al. Crosstalk between angiogenesis, cytokeratin-18, and insulin resistance in the progression of non-alcoholic steatohepatitis. World J Gastroenterol 2009;15:5193-5199.

13. Salcedo X, Medina J, Sanz-Cameno P, García-Buey L, MartínVilchez S, Moreno-Otero R. Review article: angiogenesis soluble factors as liver disease markers. Aliment Pharmacol Ther 2005;22:23-30.

14. Coulon S, Heindryckx F, Geerts A, Van Steenkiste C, Colle I, Van Vlierberghe H. Angiogenesis in chronic liver disease and its complications. Liver Int 2011;31:146-162.

15. Medina J, Arroyo AG, Sánchez-Madrid F, Moreno-Otero R. Angiogenesis in chronic inflammatory liver disease. Hepatology 2004;39:1185-1195.

16. Lai WK, Adams DH. Angiogenesis and chronic inflammation; the potential for novel therapeutic approaches in chronic liver disease. J Hepatol 2005;42:7-11.

17. Expert Panel on Detection, Evaluation, and Treatment of High Blood Cholesterol in Adults. Executive summary of the third report of the National Cholesterol Education Program (NCEP) expert panel on detection, evaluation, and treatment of high blood cholesterol in adults (Adult Treatment Panel III). JAMA 2001;285:2486-2497.

18. Tamimi TI, Elgouhari HM, Alkhouri N, et al. An apoptosis panel for nonalcoholic steatohepatitis diagnosis. J Hepatol 2011;54:1224-1229.

19. Brunt EM, Janney CG, Di Bisceglie AM, Neuschwander-Tetri BA, Bacon BR. Nonalcoholic steatohepatitis: a proposal for grading and staging the histological lesions. Am J Gastroenterol 1999;94:2467-2474.

20. Kleiner DE, Brunt EM, Van Natta $M$, et al; Nonalcoholic Steatohepatitis Clinical Research Network. Design and validation of a histological scoring system for nonalcoholic fatty liver disease. Hepatology 2005;41:1313-1321.

21. McCullough AJ. Pathophysiology of nonalcoholic steatohepatitis. J Clin Gastroenterol 2006;40 Suppl 1:S17-S29.

22. Edmison J, McCullough AJ. Pathogenesis of non-alcoholic steatohepatitis: human data. Clin Liver Dis 2007;11:75-104.

23. Machado MV, Diehl AM. Pathogenesis of nonalcoholic steatohepatitis. Gastroenterology 2016;150:1769-1777.

24. Bellentani S, Marino M. Epidemiology and natural history of non-alcoholic fatty liver disease (NAFLD). Ann Hepatol 2009;8 Suppl 1:S4-S8.

25. Sanal MG. The blind men 'see' the elephant-the many faces of fatty liver disease. World J Gastroenterol 2008;14:831-844.

26. Elpek G. Angiogenesis and liver fibrosis. World J Hepatol 2015;7:377-391.

27. Hardy T, Oakley F, Anstee QM, Day CP. Nonalcoholic fatty liver disease: pathogenesis and disease spectrum. Annu Rev Pathol 2016;11:451-496.

28. McCuskey RS, Ito Y, Robertson GR, McCuskey MK, Perry M, Farrell GC. Hepatic microvascular dysfunction during evolution of dietary steatohepatitis in mice. Hepatology 2004;40:386-393.

29. Kukla M. Angiogenesis: a phenomenon which aggravates chronic liver disease progression. Hepatol Int 2013;7:4-12.

30. Mazzanti R, Messerini L, Monsacchi L, et al. Chronic viral hepatitis induced by hepatitis $\mathrm{C}$ but not hepatitis $\mathrm{B}$ virus infection correlates with increased liver angiogenesis. Hepatology 1997;25:229-234.

31. Salcedo X, Medina J, Sanz-Cameno P, et al. The potential of 
angiogenesis soluble markers in chronic hepatitis C. Hepatology 2005;42:696-701.

32. Semela D, Dufour JF. Angiogenesis and hepatocellular carcinoma. J Hepatol 2004;41:864-880.

33. Deli G, Jin $\mathrm{CH}, \mathrm{Mu} \mathrm{R}$, et al. Immunohistochemical assessment of angiogenesis in hepatocellular carcinoma and surrounding cirrhotic liver tissues. World J Gastroenterol 2005;11:960-963.

34. Kitade M, Yoshiji H, Kojima H, et al. Leptin-mediated neovascularization is a prerequisite for progression of nonalcoholic steatohepatitis in rats. Hepatology 2006;44:983-991.

35. Aihara Y, Yoshiji H, Noguchi R, et al. Direct renin inhibitor, aliskiren, attenuates the progression of non-alcoholic steatohepatitis in the rat model. Hepatol Res 2013;43:1241-1250.

36. Yang YY, Liu RS, Lee PC, et al. Anti-VEGFR agents ameliorate hepatic venous dysregulation/microcirculatory dysfunction, splanchnic venous pooling and ascites of NASH-cirrhotic rat. Liver Int 2014;34:521-534.

37. Amarapurkar AD, Amarapurkar DN, Vibhav S, Patel ND. Angiogenesis in chronic liver disease. Ann Hepatol 2007;6:170-173.
38. Yilmaz Y, Yonal O, Kurt R, et al. Circulating levels of vascular endothelial growth factor $\mathrm{A}$ and its soluble receptor in patients with biopsy-proven nonalcoholic fatty liver disease. Arch Med Res 2011;42:38-43.

39. Coulon S, Francque S, Colle I, et al. Evaluation of inflammatory and angiogenic factors in patients with non-alcoholic fatty liver disease. Cytokine 2012;59:442-449.

40. Tarantino G, Conca P, Pasanisi F, et al. Could inflammatory markers help diagnose nonalcoholic steatohepatitis? Eur J Gastroenterol Hepatol 2009;21:504-511.

41. Ciupińska-Kajor M, Hartleb M, Kajor M, et al. Hepatic angiogenesis and fibrosis are common features in morbidly obese patients. Hepatol Int 2013;7:233-240.

42. Gill RM, Belt P, Wilson L, Bass NM, Ferrell LD. Centrizonal arteries and microvessels in nonalcoholic steatohepatitis. Am J Surg Pathol 2011;35:1400-1404.

43. Sun K, Wernstedt Asterholm I, Kusminski CM, et al. Dichotomous effects of VEGF-A on adipose tissue dysfunction. Proc Natl Acad Sci U S A 2012;109:5874-5879. 